ANNALES

UNIVERSITATIS MARIAE CURIE-SKŁODOWSKA

LUBLIN - POLONIA

VOL. LXXII, 2

SECTIO AA

2017

\title{
A review on physicochemical measurements by headspace gas chromatography. Studies of vapour-liquid equilibria
}

\author{
Andrzej Lewandowski* and Katarzyna Szymczyk \\ Department of Interfacial Phenomena, Faculty of Chemistry, \\ Maria Curie-Skłodowska University, \\ Maria Curie-Skłodowska Sq. 3, 20-031 Lublin, Poland \\ "email: andrzej.lewandowski@mail.com
}

\begin{abstract}
Headspace gas chromatography (HS-GC) is a powerful technique for the analysis of volatile compounds. It has found broad applications for quantitative and qualitative analyses of various samples as well as for physicochemical measurements. This paper briefly reviews the basic physicochemical applications of HS-GC including vapour pressure measurements and studies of vapourliquid equilibria in multicomponent systems. A special attention is paid to methodological aspects of these measurements. The advantages and limitations of HS-GC in this field as well as typical applications are also pointed out.
\end{abstract}

\section{INTRODUCTION}

Headspace gas chromatography is a group of analytical techniques that combine extraction of the analyte of interest from a condensed phase sample into a gas phase and its subsequent analysis by gas chromatography [1-3]. In general, headspace analysis can be performed in two modes: static or dynamic. In the static mode (S-HS), a liquid or solid sample containing volatile constituents is placed in a vial which after sealing is thermostated in order to reach the equilibrium distribution of volatiles between the sample and the gas phase. After equilibration, an 
aliquot of the gas phase is injected into a gas chromatograph for analysis. In the dynamic mode of headspace analysis (D-HS), continuous flow of inert gas is used to completely extract volatile constituents from the sample. The extracted volatiles are captured due to adsorption or condensation in a suitable trap and subsequently released for analysis by thermal desorption or vapourization. The dynamic headspace extraction is a non-equilibrium process. However, similar final results (exhaustive extraction of a volatile analyte from the sample) can be achieved by the equilibrium-based technique called multiple headspace extraction (MHE) which, in principle, is a dynamic gas extraction made in a stepwise mode. MHE is carried out in the way similar to the static headspace extraction. After withdrawal of an aliquot of equilibrated gas phase from a vial, a fresh portion of an inert gas is introduced into a vial and the successive steps of equilibration, extraction of gas sample and its analysis are repeated.

Headspace gas chromatography has found many applications in qualitative and quantitative analyses of volatile compounds in different types of samples. The main fields of analytical applications of headspace sampling include characterization of flavours and fragrances in food, cosmetics and plants, analysis of VOCs in the environmental samples, determination of residual solvents in pharmaceuticals and analysis of volatiles in the biological samples (blood, urine, etc.), among others [4]. Besides analytical applications, HS-GC can be also used for physicochemical measurements. The main difference between these applications, besides the fact that composition of samples used in physicochemical measurements is usually known, consists in the way of calibration and data analysis. Furthermore, due to non-equilibrium character of dynamic headspace sampling, only the S-HS and MHE techniques are used for determination of physicochemical quantities. Although some reviews on physicochemical applications of HS-GC has already been published $[1,2,5]$, significant development in this field that has taken place in the recent decade justifies the need for an updated review. In this paper, being the first part of a comprehensive review, our attention is focused on general theory of headspace sampling and on the applications related to the vapour-liquid equilibrium (VLE), including vapour pressure measurements, determination of activity coefficients in both diluted and concentrated regimes of multicomponent mixtures as well as determination of other physicochemical functions related to VLE. 


\section{BASIC THEORY OF HEADSPACE SAMPLING}

When a sample of liquid or solid containing volatile constituents is placed in a closed vial, volatile compounds distribute between the condensed (sample) and gas (headspace) phases (Fig. 1.) according to the thermodynamically controlled equilibrium [1]. If the total volume of the vial, the sample phase volume and the gas phase volume are denoted with $V_{V}, V_{S}$ and $V_{G}$, respectively, the volume balance of the system is given by equation (1):

$$
V_{V}=V_{S}+V_{G}
$$

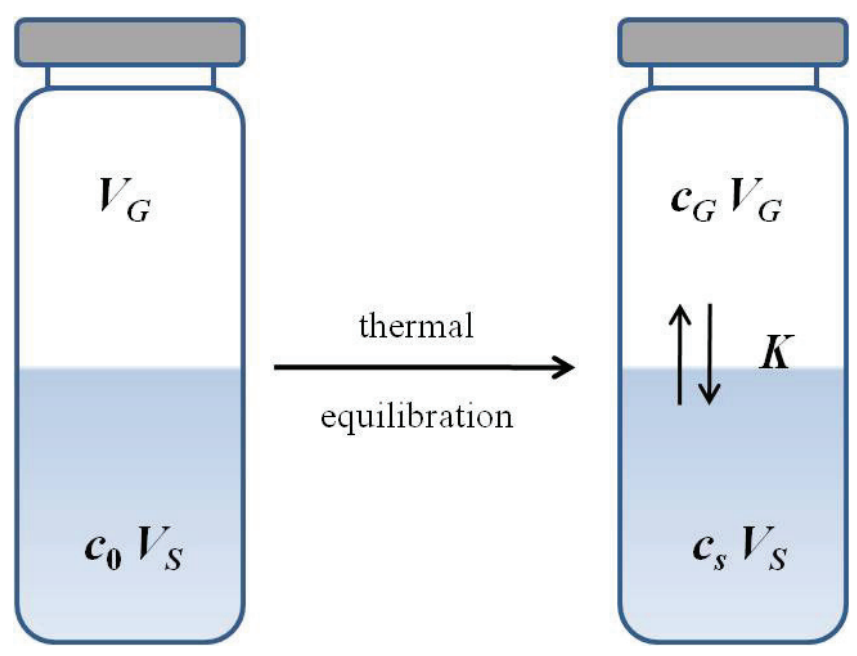

Fig.1. Distribution of a volatile analyte during the sample equilibration in the static headspace analysis (adapted from [1]).

Neglecting the change of the sample volume associated with the volatile analyte migration to the gas phase, and denoting the concentrations of the solute in the original sample and in the condensed and gas phases after reaching an equilibrium with $c_{0}, c_{S}$ and $c_{G}$, respectively, the mass balance of the analyte is given by equation (2):

$$
c_{0} \cdot V_{S}=c_{S} \cdot V_{S}+c_{G} \cdot V_{G}
$$

The ratio of concentrations of the analyte in the sample and the gas phase is called the partition (or distribution) coefficient [1,4]: 


$$
K=\frac{c_{S}}{c_{G}}
$$

The relative volumes of the two phases present in the vial are characterized by the phase ratio $\beta[1,6]$ :

$$
\beta=\frac{V_{G}}{V_{S}}
$$

Combining equations (2)-(4) gives the relation between the gas phase concentration of the analyte in equilibrium and its concentration in the original sample:

$$
c_{G}=\frac{c_{0}}{K+\beta}
$$

The peak area of the analyte resulting from a chromatographic analysis of an aliquot of equilibrated gas phase withdrawn from the vial is proportional to the concentration of the analyte in the gas phase:

$$
A=f \cdot c_{G}
$$

where the proportionality factor $f$ depends on the operational parameters of the instrument [7], including the detector response factor and the ratio of the volume of the equilibrated gas phase injected on the GC column to the total volume of the gas phase in the vial. Therefore for the defined analyte - sample matrix system at a constant temperature (a constant value of $K$ ) and with the defined analytical parameters (constant values of $f$ and $\beta$ ), the peak area for the analyte in the headspace analysis is directly proportional to the concentration of the analyte in the original sample (eq. (7)) which forms the basis of analytical applications of HS-GC [1]:

$$
A=\frac{f \cdot c_{0}}{K+\beta}
$$

According to the Raoult's law, the partial pressure of the dissolved solute $p_{i}$ over its solution is directly proportional to its mole fraction in the solution $x_{i}$ while the proportionality factor is the saturated vapour pressure of the pure substance $p_{i}^{o}[1,8]$, which fulfils the relation:

$$
p_{i}=p_{i}^{o} \cdot x_{i}
$$


The Raoult's law is valid only for the ideal solutions. In order to account for nonidealities in the liquid phase, the activity coefficient $\gamma_{i}$ [8] has to be introduced into equation (8) and we obtain the relation:

$$
p_{i}=p_{i}^{o} \cdot \gamma_{i} \cdot x_{i}
$$

Provided that the total pressure in the headspace vial during the headspace analysis is sufficiently low, the partial pressure of the solute can be related to the molar concentration of the solute in the gas phase using the ideal gas law:

$$
c_{G(i)}=\frac{n_{G(i)}}{V_{G}}=\frac{p_{i}}{R \cdot T}=\frac{p_{i}^{o}}{R \cdot T} \cdot \gamma_{i} \cdot x_{i}
$$

Introducing eq. (10) into eq. (6) yields:

$$
A_{i}=\frac{f_{i} \cdot p_{i}^{o}}{R \cdot T} \cdot \gamma_{i} \cdot x_{i}
$$

Equation (11) shows that the peak area of the analyte is a linear function of the molar fraction of the analyte in solution if the activity coefficient is constant in the studied range of concentrations. This holds true only for the diluted solutions when the solute molecules are surrounded by solvent molecules and only the solute-solvent interactions come into play. For more concentrated solutions, the probability of solute-solute interactions increases and the activity coefficient becomes a function of solute concentration [1]. In the case of pure substances both the molar fraction of the analyte and its activity coefficient equal 1 . The peak area is then directly proportional to the vapour pressure of the substance.

\section{DETERMINATION OF VAPOUR PRESSURES}

The application of headspace analysis for determination of the vapour pressure was firstly reported by Leggett [9]. Excessive amounts of 2,4,6,-trinitrotoluene (TNT) were placed in tightly sealed vials and thermostated in a water bath for 2-4 weeks. The equilibrated vapour samples were withdrawn using a gas-tight syringe and analyzed by a gas chromatograph equipped with an electron capture detector (ECD). Since the volume of vapour withdrawn from the vial was exactly known, the vapour pressure was calculated using the ideal gas law from the obtained 
peak area and the detector response factor. Due to high sensitivity of $\mathrm{ECD}$, the vapour pressure as low as $0.27 \cdot 10^{-6} \mathrm{~mm} \mathrm{Hg}$ could be determined for TNT at $12^{\circ} \mathrm{C}$ [9].

In the case of automated headspace sampling, the volume of the equilibrated vapour used for chromatographic analysis (a fraction of the gas phase volume withdrawn from a vial) depends on sampling parameters of the instrument and usually is not exactly known. Therefore appropriate calibration techniques have to be employed in order to correlate the peak area with the vapour pressure of the test substance. One approach is to prepare a series of vapour phase standards to relate the peak area with the gas phase concentration [10]. A series of vials is filled with different amounts of test substance, sealed and analyzed. The amounts added to the vials should be low enough to ensure complete vaporization at the test temperature. Subsequently, the analysis of the vial containing an excess of a test substance allows to determine gas phase concentration of saturated vapour. For the substances with low vapour pressure it can be difficult to prepare the calibration curve due to difficulties in accurate weighing of small amounts of the test substance. In these cases vapour phase standards could be prepared from appropriate solutions of the test substance in a more volatile solvent, provided that: (i) the desired different amounts of the test substance are introduced to the vials as the same volume of solutions with different concentrations of the solute; (ii) both solute and solvent will vaporize completely at the test temperature; (iii) solvent and solute will be well separated on the column. Although standards with low amounts of the test substance could be prepared using diluted solutions, adsorption of the test substance on the walls of the vial or in the sampling path often makes it impossible to establish a reliable calibration curve in the range of low gas phase concentrations $[1,10]$. Therefore the lower determination limit of the method using the vapour phase calibration falls in the range of 1-10 $\mathrm{Pa}$ $[10,11]$.

Schoene et al. [11] have extended this limit down to $0.01 \mathrm{~Pa}$ using a conceptually different calibration procedure. The proposed method consists in: (i) determination of the mass of the test substance injected from the vial filled with saturated vapour; (ii) determination of the corresponding volume of the saturated vapour injected on the column. Firstly, a calibration curve should be prepared relating the peak area to the exact amount of the test substance injected on the column. This can be done by simple liquid injections of a defined volume of solutions with different concentrations of the test substance. Once the calibration curve 
is obtained, the mass of the test substance injected from the headspace vial saturated with its vapour can be calculated. The same steps are repeated with a reference compound of known vapour pressure at the test temperature. This allows calculation of the volume of the saturated vapour injected on the column, since both the mass injected and the gas phase concentration of saturated vapour can be determined from the calibration curve and the ideal gas law, respectively. Provided that the operational parameters of the headspace sampler are kept identical for both the test substance and the reference compound, the saturation gas phase concentration of the test substance can be calculated from the injected mass and volume. The vapour pressure of the test substance can be calculated using the ideal gas law.

The measurements of vapour pressure by HS-GC ara particularly useful for the compounds of low volatility or for low temperature measurements. Another advantage of this technique is that the studied compounds do not need to be highly purified, as minor contaminants could be separated from the compound of interest during the chromatographic analysis. Furthermore, the amount of substance needed for the analysis is very small and the measurements in the closed vials help avoid emission of dangerous substances into the environment. Therefore the headspace analysis has found applications for vapour pressure measurements of such substances as chlorinated compounds [11], fuels [12, 13] and especially explosives [9, 14, 15].

\section{STUDIES OF VAPOUR-LIQUID EQUILIBRIA}

According to equation (11) the headspace analysis of the pure substance gives the peak area which is directly proportional to the vapour pressure at the equilibration temperature. Therefore at the specified temperature, the peak area of the analyte in the multicomponent system will be proportional to the product of molar fraction of the solute and its activity coefficient, with the peak area obtained for the pure substance $A_{i 0}$ as the proportionality factor, provided that the remaining analytical parameters (such as phase ratio) remain unchanged [16]:

$$
A_{i}=A_{i 0} \cdot \gamma_{i} \cdot x_{i}
$$

From equation (12) it is possible to calculate the value of the solute activity in the multicomponent mixture if its molar fraction is known: 


$$
\gamma_{i}=\frac{A_{i}}{A_{i 0} \cdot x_{i}}
$$

For the sufficiently small value of the phase ratio (i.e. relatively large amount of the sample in the vial) and low vapour pressure of the studied components, the change of the sample composition resulting from evaporation of its components into the gas phase can be neglected and the composition of the liquid phase in equilibrium with the gas phase can be assumed to be identical to that of the liquid sample added to the vial. Equation (13) forms the basis of a simple and straightforward measurement of activity coefficients in the multicomponent liquid mixtures by static headspace chromatography. The values of the activity coefficient determined at specified compositions of liquid mixtures can be used to determine other thermodynamic functions of the liquid mixture, such as partial free molar energy of mixing or total free energy of mixing [16].

The headspace analysis can be also used for the direct measurements of the gas phase composition of the studied system. In the case of the binary mixture, the molar ratio of components in the gas phase can be calculated from equation:

$$
\frac{y_{1}}{y_{2}}=\frac{A_{1}}{A_{2}} \cdot \frac{f_{2}}{f_{1}}
$$

where $y_{i}$ stands for the molar fraction of the $i$-th component in the gas phase [17]. In order to facilitate this calculation the ratio of response factors has to be determined separately. This can be easily calculated from the peak area ratio resulting from the $\mathrm{GC}$ analysis of the equimolar liquid mixture of the studied components.

Reliable measurements of the activity coefficients for the concentrated mixtures of volatile substances require standardization of the analytical parameters, such as phase ratio and equilibration temperature [17]. The tests of thermodynamic consistency of the obtained results should be also taken to validate reliability of the obtained data [18].

The main advantages of the headspace analysis for the studies of vapour-liquid equilibrium stem from the possibility of simultaneous measurements of gas phase concentrations of all components of the studied multicomponent system and from the possibility of measurements automation. Therefore the headspace analysis has been widely employed to study the vapour-liquid equilibrium of both binary $[16,19-24]$ and ternary $[18,22,23,25-27]$ systems. 


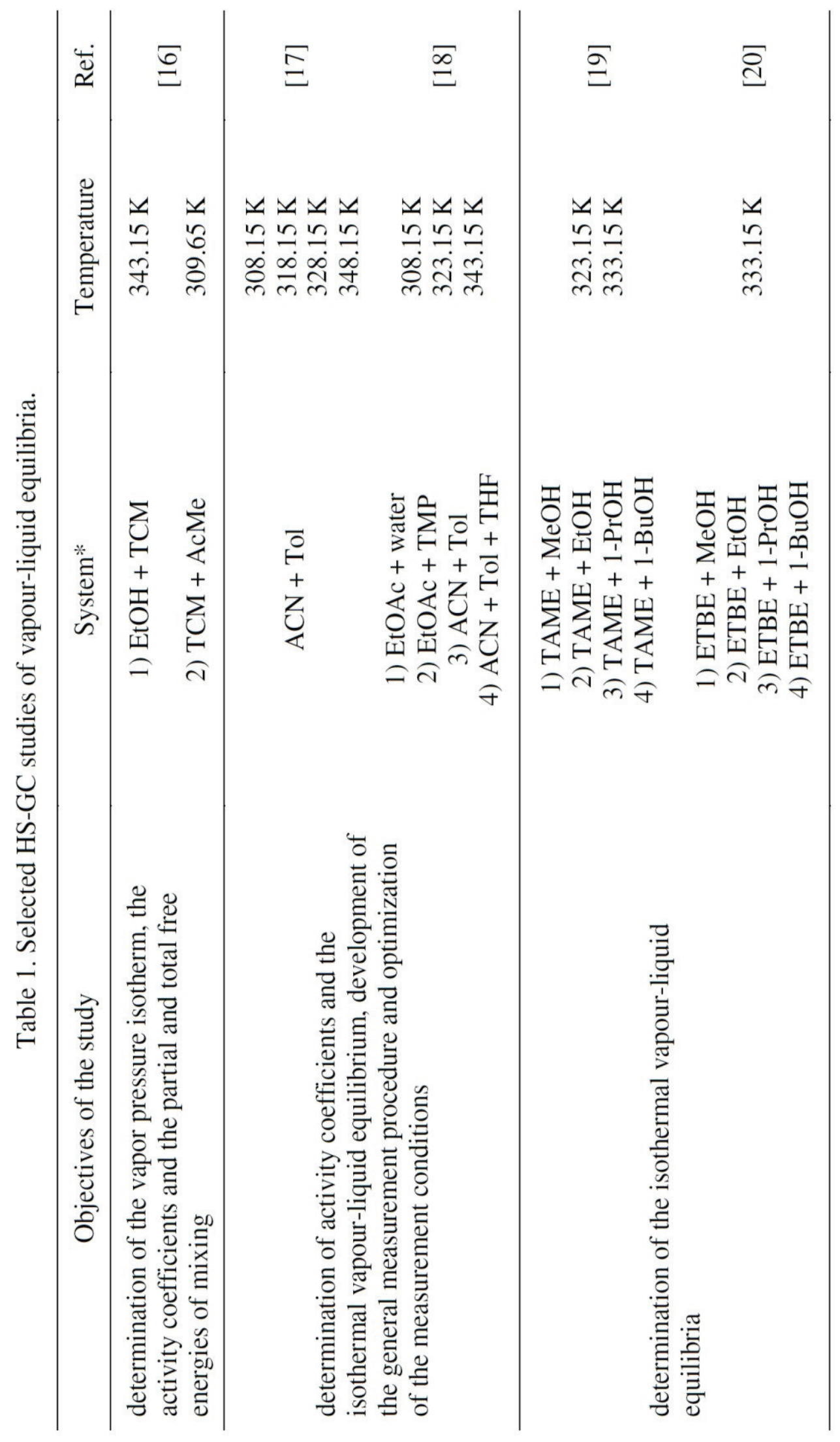




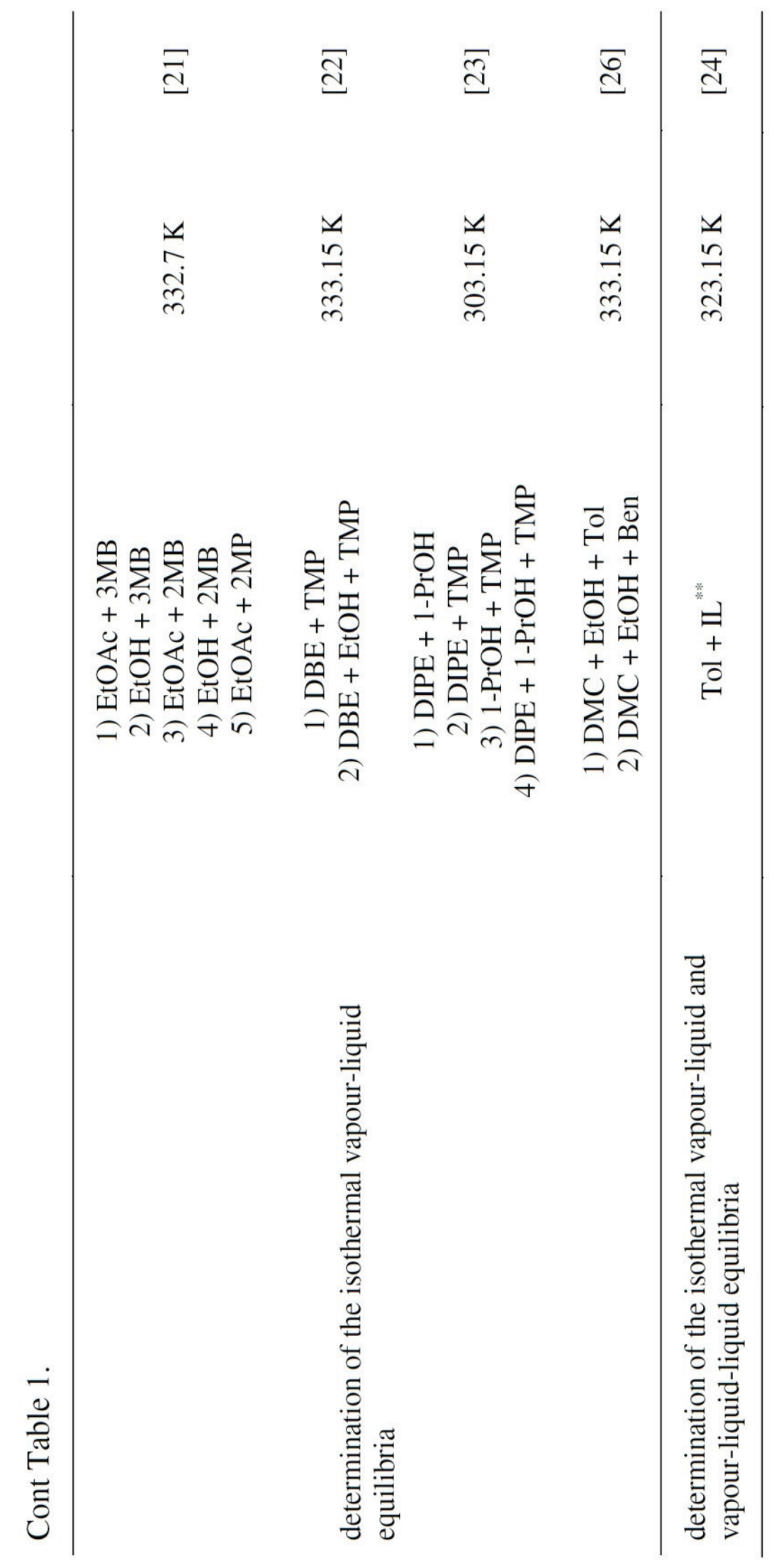




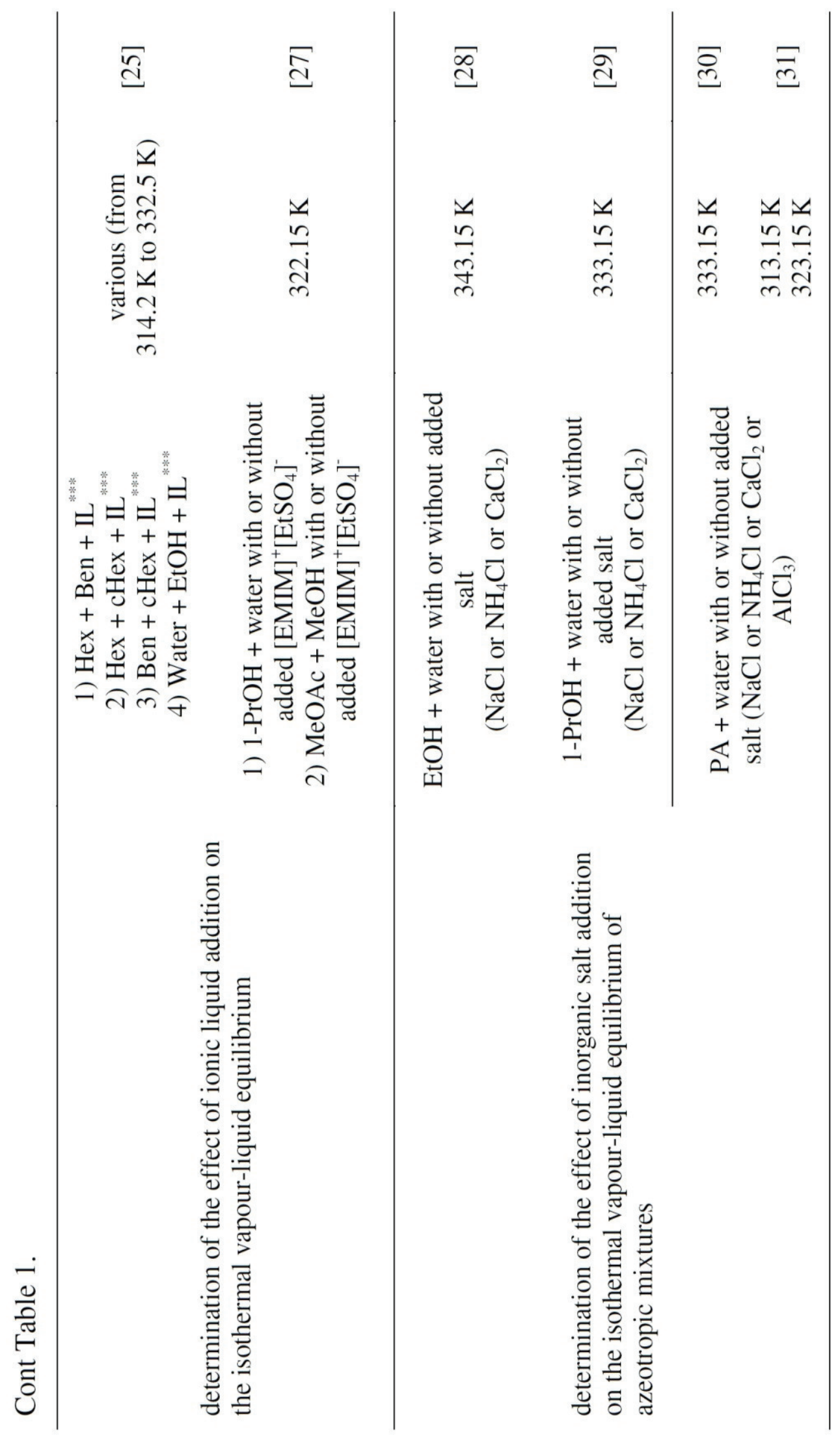




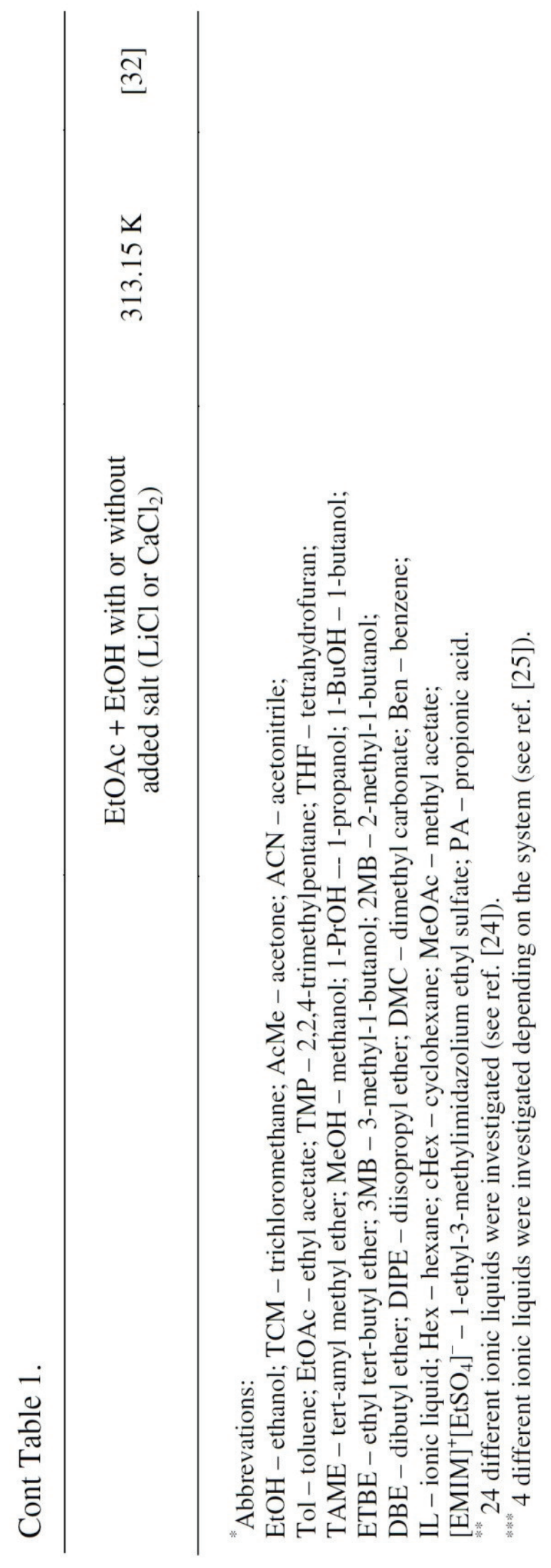


A special attention of researchers was focused on the effects of ionic liquids [24, 25, 27] and simple electrolytes [28-32] addition on the vapour-liquid equilibria, in search for improvement of separation of azeotropic mixtures by distillation. The objectives of selected headspace gas chromatographic studies of VLE, together with the details on the investigated systems are given in Table 1.

\section{CONCLUSIONS}

Due to high availability of automated headspace samplers for gas chromatography this analytical technique has found many applications both in analytical and physical chemistry. Owing to high sensitivity and a wide linearity range of GC detectors as well as possibility of analyzing several compounds in a single GC run, it can be used for physicochemical measurements of both single- and multi-component systems with a wide range of concentrations.

\section{REFERENCES}

[1] B. Kolb and L. S. Ettre, Static Headspace-Gas Chromatography: Theory and Practice, 2nd ed., Wiley-Interscience, Hoboken, 2006.

[2] B. V. Ioffe and A. G. Vitenberg, Head-Space Analysis and Related Methods in Gas Chromatography, Wiley-Interscience, New York, 1984.

[3] H. Hachenberg and A. P. Schmidt, Gas Chromatographic Headspace Analysis, Heyden, London, 1977.

[4] A. C. Soria, M. J. García-Sarrió, and M. L. Sanz, Trends Anal. Chem. 71, 85 (2015).

[5] J. Y. Zhu and X. S. Chai, Curr. Anal. Chem. 1, 79 (2005).

[6] L. S. Ettre, C. Welter, and B. Kolb, Chromatographia 35, 73 (1993).

[7] N. H. Snow and G. P. Bullock, J. Chromatogr. A 1217, 2726 (2010).

[8] P. Atkins and J. De Paula, Atkins' Physical Chemistry, 8th ed., W. H. Freeman, New York, 2006.

[9] D. C. Leggett, J. Chromatogr. 133, 83 (1977).

[10] K. Schoene and J. Steinhanses, Z. Anal. Chem. 309, 198 (1981).

[11] K. Schoene, W. Böhmer, and J. Steinhanses, Z. Anal. Chemie 319, 903 (1984).

[12] J. E. Woodrow and J. N. Seiber, J. Chromatogr. 455, 53 (1988).

[13] J. E. Woodrow, Energy Fuels 17, 216 (2003).

[14] J. C. Oxley, J. L. Smith, K. Shinde, and J. Moran, Propellants, Explos. Pyrotech. 30, 127 (2005). 
[15] J. C. Oxley, J. L. Smith, W. Luo, and J. Brady, Propellants, Explos. Pyrotech. 34, 539 (2009).

[16] B. Kolb, J. Chromatogr. 112, 287 (1975).

[17] P. Luis, C. Wouters, B. Van der Bruggen, and S. I. Sandler, J. Chromatogr. A 1302, 111 (2013).

[18] P. Luis, C. Wouters, N. Sweygers, C. Creemers, and B. Van Der Bruggen, J. Chem. Thermodyn. 49, 128 (2012).

[19] J.-H. Oh and S.-J. Park, J. Chem. Eng. Data 42, 517 (1997).

[20] J.-H. Oh and S.-J. Park, J. Chem. Eng. Data 43, 1009 (1998).

[21] M. T. Sanz and J. Gmehling, Fluid Phase Equilib. 267, 158 (2008).

[22] J.-Y. Lee, I.-C. Hwang, S.-J. Park, and S.-J. In, J. Chem. Eng. Data 55, 864 (2010).

[23] I.-C. Hwang, M.-Y. Jo, H.-Y. Kwak, S.-J. Park, and K.-J. Han, J. Chem. Eng. Data 52, 2503 (2007).

[24] E. J. González, J. Palomar, P. Navarro, M. Larriba, J. García, and F. Rodríguez, J. Mol. Liq. 250, 9 (2018).

[25] B. Mokhtarani and J. Gmehling, J. Chem. Thermodyn. 42, 1036 (2010).

[26] I.-C. Hwang, S.-J. Park, and K.-J. Han, Fluid Phase Equilib. 303, 150 (2011).

[27] H. Matsuda, K. Tochigi, V. Liebert, and J. Gmehling, Fluid Phase Equilib. 307, 197 (2011).

[28] F. A. Banat, F. A. Abu Al-rub, and J. Simandl, Chem. Eng. Technol. 22, 761 (1999).

[29] F. A. Abu Al-Rub, F. A. Banat, and J. Simandl, Chem. Eng. J. 74, 205 (1999).

[30] F. Banat, S. Al-Asheh, and J. Simandl, Chem. Eng. Process. 41, 793 (2002).

[31] F. Banat, S. Al-Asheh, and J. Simandl, Chem. Eng. Process. Process Intensif. 42, 917 (2003).

[32] H. Takamatsu and S. Ohe, J. Chem. Eng. Data 48, 277 (2003). 